\title{
Dose-Delivery Time Interval of Morphine in Labour and its Impact on the Likelihood of Adverse Neonatal Outcomes
}

\author{
Ranatunga $M$ (D) and Doctor TN* \\ ${ }^{1}$ Medical Student, Melbourne University, Melbourne, Australia \\ ${ }^{2}$ General Pediatrician and Neonatologist, The Northern Hospital, Epping, Australia
}

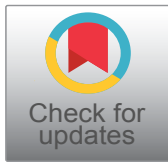

*Corresponding author: Tejas Doctor N, General pediatrician and Neonatologist, The Northern Hospital, Epping, Australia

\begin{abstract}
Background: To find the effect that time between dosage of morphine and delivery of the baby has on the incidence of need for neonatal resuscitation.

Methods: Retrospective analysis of 659 births at The Northern Hospital, Epping, Victoria, featuring use of morphine in labour. Time between morphine dose and delivery of baby was recorded for each birth, known as the dose-delivery interval (DDI). Four equally distributed categories were created based on quartiles of DDI. Various neonatal outcomes were compared between the groups, including need for resuscitation, and type of resuscitation used. Univariate and multivariate analysis were performed using IBM SPSS version 26.
\end{abstract}

Results: Dose-delivery category 2 (155-314 minutes) was found to have significantly higher odds of requiring neonatal resuscitation (OR 2.08; 95\% Cl 1.20-3.59 $\mathrm{P}=$ 0.009 ), in addition to specific forms of resuscitation such as tactile stimulation (OR 2.05; 95\% Cl 1.20-3.51 P = 0.009), continuous positive airway pressure (OR $2.51 ; 95 \% \mathrm{Cl} 1.20$ $5.25 \mathrm{P}=0.015)$ and intermittent positive pressure ventilation (OR 6.67; 95\% Cl 2.53-17.62 P $<0.001$ ) compared to the longest dose-delivery category (> 526.5 minutes). Subcutaneous morphine was also found to have lower odds of neonatal resuscitation (OR 0.22; 95\% Cl 0.068-0.69 P = 0.009 ) and specifically tactile stimulation (OR $0.24 ; 95 \% \mathrm{Cl}$ 0.08-0.77 $\mathrm{P}=0.016$ ) compared to intramuscular morphine.

Conclusion: A dose-delivery interval between 155 and 314 minutes may lead to a higher incidence of requirement for resuscitation at birth, in addition to specific types of resuscitation including tactile stimulation, continuous positive airway pressure and intermittent positive pressure ventilation.

\section{Keywords}

Morphine, Pregnancy, Labour, Neonates

\section{Introduction}

Parenteral opioids are a commonly used form of analgesia available to women in labour worldwide [1]. The most commonly used systemic analgesia in labour include agonist opioids such as morphine, fentanyl, remifentanil and pethidine or agonist-antagonist opioids such as nalbuphine and butorphanol [1]. Opioids in labour have been found to be associated with maternal, fetal and neonatal side effects. The most concerning maternal side effect is respiratory depression, others include sedation, nausea/vomiting, dizziness, altered mental status, decreases gastric mobility/emptying and urinary retention [2]. In the neonate, placental transfer of opioids such as pethidine may result in respiratory depression and subtle neurobehavioral changes, which may manifest as delays in breastfeeding [3].

An important point of consideration when observing the incidence of adverse neonatal outcomes is the time interval between opioid dose and delivery of the baby, referred to as the dose-delivery interval (DDI). While DDI observation and correlation with adverse neonatal outcomes has been commonly featured in research involving other intrapartum opioids such as pethidine, there is an apparent deficiency in similar research involving morphine.

Current literature relating to the intrapartum use of morphine is mainly in the form of randomised control trials comparing morphine or diamorphine to pethidine. A single other study reports on diamorphine and the

Citation: Ranatunga M, Doctor TN (2021) Dose-Delivery Time Interval of Morphine in Labour and its Impact on the Likelihood of Adverse Neonatal Outcomes. Int J Pediatr Res 7:084. doi.org/10.23937/24695769/1510084

Accepted: December 24, 2021: Published: December 26, 2021

Copyright: (c) 2021 Ranatunga M, et al. This is an open-access article distributed under the terms of the Creative Commons Attribution License, which permits unrestricted use, distribution, and reproduction in any medium, provided the original author and source are credited. 
dose-delivery interval, cord venous blood concentration of free morphine and neonatal outcomes [4]. A majority of literature up to date has demonstrated no significant differences between pethidine and morphine with regards to neonatal outcomes, other than one study which found fewer incidences of Apgar score less than seven in IM diamorphine compared to IM pethidine [5].

The primary aim of our study is to observe the impact the time between morphine dose and delivery of the baby has on the incidence of both early and late indicators of neonatal outcomes. We hypothesize that a reduced dose-delivery interval of morphine predisposes to an increased risk of adverse neonatal outcomes compared to longer dose-delivery intervals.

\section{Methods}

\section{Study design}

Retrospective cohort study (Figure 1).

\section{Setting}

Research was based on all births at The Northern Hospital (Epping) between the $1^{\text {st }}$ December 2018 to $1^{\text {st }}$ December 2019. Data was obtained through the Birth Outcomes System (BOS) program. This yielded 3593 births in this time period. The medication charts for all these births were manually screened to exclude births that did not feature morphine as a form of labour analgesia, yielding a dataset of 659 births. Data was deidentified and recorded manually on Microsoft Excel.

\section{Participants}

Inclusion criteria: Morphine use in labour, regardless of route of administration or amount given.

Exclusion criteria: Gestational age < 30 weeks, neonatal mortality before labour (stillbirths). Babies born with severe congenital malformation. Data containing unclear documentation.

\section{Variables}

Outcomes: Several neonatal outcomes were observed; including need for resuscitation, type of resuscitation used.

Exposures: Primary exposure observed was the duration between morphine dose and delivery of the baby (dose-delivery interval/DDI). Other main exposures included route of morphine dose (intramuscular/intravenous/subcutaneous) and total amount of morphine.

Confounding variables that were recorded were separated into maternal and neonatal variables. Maternal confounding variables included maternal age, maternal country of birth, gravidity, parity, gestational age, mode of delivery and additional analgesia given in labour such as epidural, spinal anaesthetic, intravenous fentanyl, intramuscular pethidine. Neonatal confounding variables included gender and birthweight.

\section{Procedure}

Initial data collection was based on manual checking

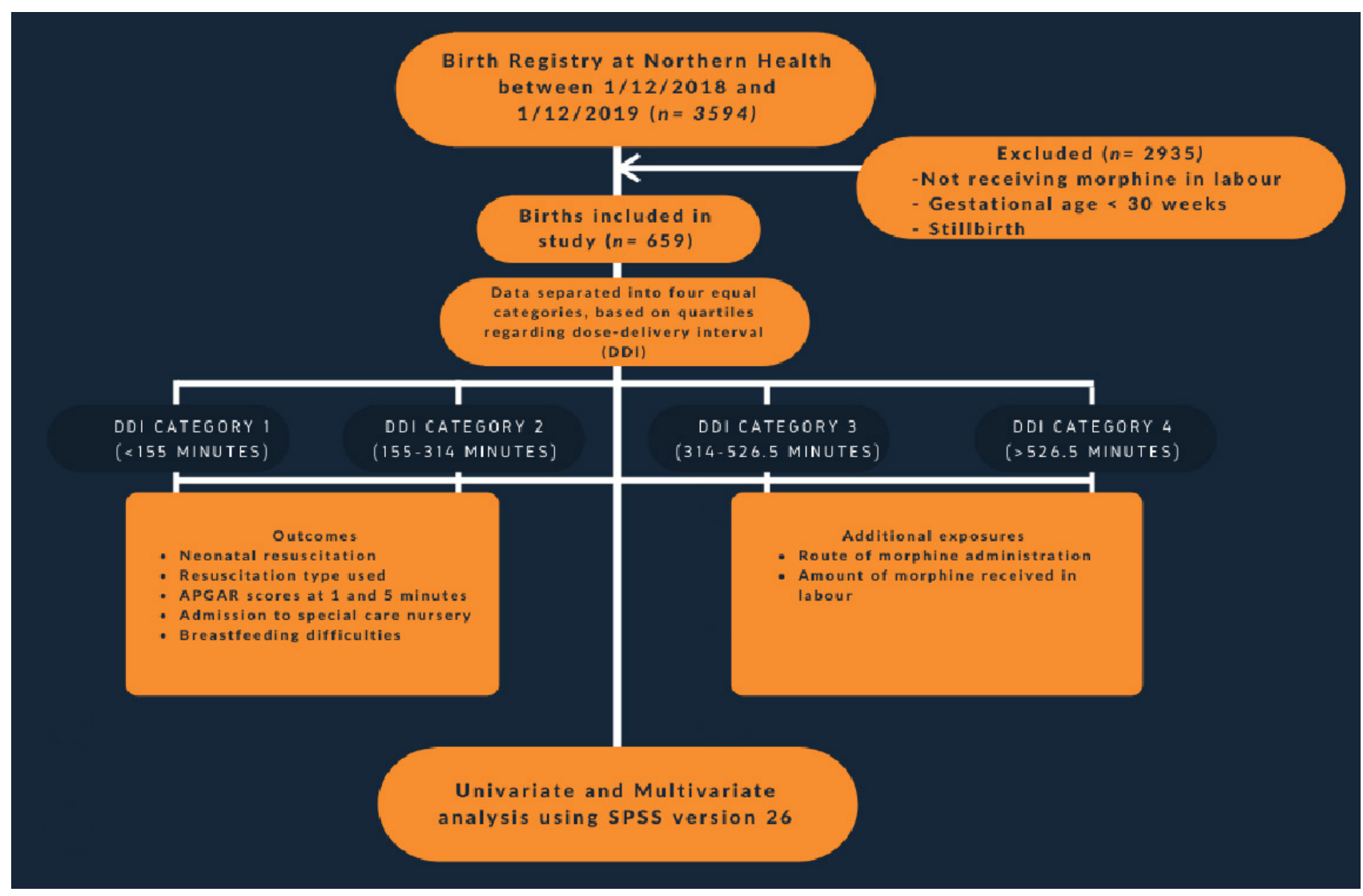

Figure 1: Retrospective cohort study. 
of each individual birth record obtained from the birth registry between $1 / 12 / 2018$ to $1 / 12 / 2019$. Each birth record was manually checked, firstly by viewing the medication chart for evidence of morphine use in the labour. If morphine were not included, the birth would be excluded from the research with no further variables recorded. If morphine use was featured, the route of administration, amount of morphine in milligrams and time of administration were all initially recorded based on the medication record. Following this, other variables were manually recorded dependent on the patient file, obstetric discharge summary and neonatal patient file. Additional information regarding neonatal resuscitation methods, additional analgesia, maternal demographics and SCN admission were also made available by a report generated by the Birth Outcomes System (BOS), obtained by consultation with the Health Information Manager at The Northern Health. This study had gone through the ethics committee and was approved by them.

Recording of outcomes: Neonatal resuscitation was recorded as a binary variable, which included all resuscitation methods. Types of neonatal resuscitation used were then also listed as binary variables in separate columns, these included tactile stimulation, suction, continuous positive airway pressure (CPAP), intermittent positive pressure ventilation (IPPV), oxygen therapy, IV dextrose, volume expander and external cardiac massage.

Recording of exposures: Dose-delivery interval was calculated via calculating the difference between time of birth and time of last morphine administration. This was recorded initially in the continuous format as minutes. After the initial data collection was done, dose-delivery interval (DDI) categories were formed based on calculation of quartiles. Four categories with equal numbers were then formed, and each birth was then assigned to a DDI category. With regards to route of morphine administration, this was recorded in the categorical format, in one of three categories (intramuscular, intravenous, and subcutaneous. Total amount of morphine was recorded as milligrams, in the continuous format by finding the cumulative amount of morphine received throughout the entire labour. If a dosage of morphine was listed as a range, i.e. '5-10 mg' was treated as a missing data entry due to lack of clarity.

\section{Recording of confounding variables}

Maternal variables such as maternal age, gravidity and parity were recorded in the continuous format. Gestational age was also recorded as a continuous variable, however, was converted to a decimal format for ease of later data analysis. Maternal country of origin was recorded in the categorical format as one of 8 categories dependent on geographical location. Mode of delivery was recorded as one of three categories: normal vaginal delivery, caesarean or instrumental.
Neonatal gender was recorded as a binary variable. Neonatal birthweight was recorded as a continuous variable.

\section{Statistical analysis}

IBM SPSS version 26 was used for the entire statistical analysis. A Biostatistician was consulted through the Northern Clinical School.

Descriptive statistics (Univariate analysis): Univariate analysis was performed first, observing associations between all variables and the primary exposure (dose-delivery interval category).

Continuous variables were tested for normality via the Shapiro-Wilk test, which found all continuous variables were skewed except for birthweight which was found to be normally distributed. Univariate analysis was then performed for DDI category against skewed continuous variables through non-parametric testing. Kruskal Wallis 1-way ANOVA was used for testing skewed variables such as maternal age, gravidity, parity, total amount of morphine, gestational age,. Parametric testing (One-Way ANOVA) was used for birthweight as it was normally distributed.

Pearson chi-square test was used for univariate analysis of DDI category against other binary or categorical variables observed in the study. Fischer exact test was used instead if cell counts were less than five.

From this initial process, four significant outcomes were obtained: neonatal resuscitation, tactile stimulation, continuous positive air way pressure (CPAP) and intermittent positive pressure ventilation (IPPV). Further univariate analysis was performed using these binary variables, to find associations with other exposures. Pearson chi-square tests and Fischer exact tests were used.

Non-parametric testing (Mann-Whitney (rank-sum) test) was used for univariate analysis of these binary outcomes against the skewed continuous variables. Parametric testing (Student's T-test) was used for univariate analysis against birthweight.

Missing data: Data that could not be obtained at all through browsing of health records or BOS reports were separately coded and excluded from data analysis.

With regards to route of administration, if multiple routes were listed e.g. 'IM/IV, IM/SC etc', these were coded separately and excluded from final data analysis due to lack of clarity.

Multivariable analysis: Outcomes which were found to be significantly associated with DDI category in univariate analysis such as neonatal resuscitation, tactile stimulation, CPAP, IPPV were considered.

Multivariable analysis was performed using manual backwards stepwise regression. Outcomes considered 
were those that were found to be significant in univariate analysis. Binary logistic regression analysis was used, with the initial model including exposures which were clinically known to be associated with the outcome or had a p-value less than 0.2 in univariate analysis with the binary outcome.

The initial models included the binary outcome of interest as the dependent variable in addition to the following exposure variables as predictors; dose delivery interval category, type of delivery, maternal age, maternal country of origin, route of morphine administration, amount of morphine administered during labour, epidural, spinal anaesthetic, intravenous fentanyl, intramuscular pethidine, baby gender, gestational age, gravidity, parity and birthweight.

The reference category was set to be the longest

Table 1: Baseline maternal characteristics by dose-delivery interval $(n=659)$.

\begin{tabular}{|c|c|c|c|c|c|}
\hline & DDI category 1 & DDI category 2 & DDI category 3 & DDI category 4 & P-value \\
\hline Maternal characteristics & $\begin{array}{l}<155 \text { mins } \\
(n=166)\end{array}$ & $\begin{array}{l}155-314 \text { mins } \\
(n=164)\end{array}$ & $\begin{array}{l}314-526.5 \mathrm{mins} \\
(\mathrm{n}=165)\end{array}$ & $\begin{array}{l}>526.5 \text { mins } \\
(n=164)\end{array}$ & \\
\hline Maternal age (years), median (IQR) & $29(25-33)$ & $29(25-33)$ & $29(25-32)$ & $28(26-32)$ & 0.495 \\
\hline Parity, n (\%) & $1(0-2)$ & $1(0-1)$ & $0(0-0)$ & $0(0-0)$ & $<0.001$ \\
\hline Gravidity, n (\%) & $2(1-3)$ & $2(1-3)$ & $1(1-2)$ & $1(1-2)$ & $<0.001$ \\
\hline Maternal country of birth, $n(\%)$ & & & & & 0.287 \\
\hline Oceania & $83(50.0 \%)$ & $73(44.5 \%)$ & $74(44.8 \%)$ & $78(47.6 \%)$ & \\
\hline Asia & $52(31.3 \%)$ & $60(36.6 \%)$ & $59(35.8 \%)$ & $61(37.2 \%)$ & \\
\hline Middle East & $22(13.3 \%)$ & $24(14.6 \%)$ & $23(13.9 \%)$ & $13(7.9 \%)$ & \\
\hline Eastern Europe & $5(3.0 \%)$ & $2(1.2 \%)$ & $2(1.2 \%)$ & $5(3.0 \%)$ & \\
\hline European Union & $0(0.0 \%)$ & $0(0.0 \%)$ & $3(1.8 \%)$ & $2(1.2 \%)$ & \\
\hline Africa & $3(1.8 \%)$ & $3(1.8 \%)$ & $0(0.0 \%)$ & $4(2.4 \%)$ & \\
\hline The Caribbean & $0(0.0 \%)$ & $0(0.0 \%)$ & $0(0.0 \%)$ & $1(0.6 \%)$ & \\
\hline North American & $1(0.6 \%)$ & $0(0.0 \%)$ & $1(0.6 \%)$ & $0(0.0 \%)$ & \\
\hline Not stated & $0(0.0 \%)$ & $2(1.2 \%)$ & $3(1.8 \%)$ & $0(0.0 \%)$ & \\
\hline Mode of delivery, $n(\%)$ & & & & & $<0.001$ \\
\hline Normal vaginal delivery & $140(84.3 \%)$ & $103(62.8 \%)$ & $67(40.6 \%)$ & $40(24.4 \%)$ & \\
\hline Caesarean & $13(7.8 \%)$ & $33(20.1 \%)$ & $48(29.1 \%)$ & $69(42.1 \%)$ & \\
\hline Instrumental delivery & $13(7.8 \%)$ & $28(17.1 \%)$ & $50(30.3 \%)$ & $55(33.5 \%)$ & \\
\hline Epidural, n (\%) & & & & & $<0.001$ \\
\hline No & $156(93.9 \%)$ & $124(76.5 \%)$ & $67(40.9 \%)$ & $35(21.3 \%)$ & \\
\hline Yes & $10(6.1 \%)$ & $38(23.5 \%)$ & $97(59.1 \%)$ & $129(78.7 \%)$ & \\
\hline Spinal anaesthetic, n (\%) & & & & & 0.184 \\
\hline No & $165(99.4 \%)$ & $156(96.3 \%)$ & $159(97.0 \%)$ & $161(98.2 \%)$ & \\
\hline Yes & $1(0.6 \%)$ & $6(3.7 \%)$ & $5(3.0 \%)$ & $3(1.8 \%)$ & \\
\hline Fentanyl PCA, n (\%) & & & & & 0.182 \\
\hline No & $166(100.0 \%)$ & $162(100.0 \%)$ & $164(100.0 \%)$ & $162(98.8 \%)$ & \\
\hline Yes & $0(0.0 \%)$ & $0(0.0 \%)$ & $0(0.0 \%)$ & $2(1.2 \%)$ & \\
\hline IM pethidine, $\mathrm{n}(\%)$ & & & & & 1.000 \\
\hline No & $165(99.4 \%)$ & $162(100.0 \%)$ & $164(100.0 \%)$ & $164(100.0 \%)$ & \\
\hline Yes & $1(0.6 \%)$ & $0(0.0 \%)$ & $0(0.0 \%)$ & $0(0.0 \%)$ & \\
\hline $\begin{array}{l}\text { Route of morphine administration, } \\
\text { n (\%) }\end{array}$ & & & & & 0.816 \\
\hline Intramuscular (IM) & $145(87.3 \%)$ & $140(85.4 \%)$ & $140(84.8 \%)$ & $150(91.5 \%)$ & \\
\hline Intravenous (IV) & $3(1.8 \%)$ & $1(0.6 \%)$ & $3(1.8 \%)$ & $4(2.4 \%)$ & \\
\hline Subcutaneous (SC) & $6(3.6 \%)$ & $7(4.3 \%)$ & $4(2.4 \%)$ & $4(2.4 \%)$ & \\
\hline $\begin{array}{l}\text { Total amount of morphine, median } \\
\text { (IQR) }\end{array}$ & $10(10-10)$ & $10(10-10)$ & $10(10-10)$ & $10(10-10)$ & 0.051 \\
\hline
\end{tabular}


DDI category (> 526.50 minutes), manual backwards stepwise regression was performed until all variables remaining in the model were significant.

\section{Results}

Baseline characteristics were comparable between the four dose-delivery interval categories (Table 1 and Table 2).

Significant differences were found with regards to the following maternal characteristics; gravidity, parity, mode of delivery and use of epidural when comparing the four dose-delivery interval categories (Table 1). With regards to neonatal characteristics, significant differences were found with regards to gestational age median, birthweight mean, need for resuscitation, need for tactile stimulation, need for continuous positive airway pressure (resuscitation) and need for intermittent positive airway pressure ventilation (resuscitation) (Table 2).

\section{Primary outcomes}

Neonates in the second dose-delivery interval (DDI) category (155-314 minutes) had an increased requirement for resuscitation at birth, also observed in specific resuscitation methods such as tactile stimulation, continuous positive airway pressure and intermittent positive pressure ventilation (Table 3). These results were observed with using the longest (fourth) DDI category (> 526.5 minutes) as the reference category.

\section{Neonatal resuscitation}

Neonates born in the second DDI category had a 2 -fold increase in odds of requirement for resuscitation compared to the longest DDI category (OR 2.08; 95\% Cl 1.20-3.59 $\mathrm{P}=0.009)$. There were no statistically significant increases in odds in either the first $(P=0.46)$ or third $(P=0.92) D D I$ category compared to the longest

Table 2: Baseline neonatal characteristics by dose-delivery interval $(n=659)$.

\begin{tabular}{|c|c|c|c|c|c|}
\hline Neonatal characteristics & $\begin{array}{l}\text { DDI category } 1 \\
<155 \text { mins } \\
(n=166)\end{array}$ & $\begin{array}{l}\text { DDI category } 2 \\
155-314 \text { mins } \\
(n=164)\end{array}$ & $\begin{array}{l}\text { DDI category } 3 \\
314-526.5 \text { mins } \\
(n=165)\end{array}$ & $\begin{array}{l}\text { DDI category } 4 \\
>526.5 \text { mins } \\
(n=164)\end{array}$ & P-value \\
\hline Gender & & & & & 0.814 \\
\hline Male & $82(49.4 \%)$ & $79(48.8 \%)$ & $77(47.0 \%)$ & $73(44.5 \%)$ & \\
\hline Female & $84(50.6 \%)$ & $83(51.2 \%)$ & $87(53.0 \%)$ & $91(55.5 \%)$ & \\
\hline $\begin{array}{l}\text { Gestational age (weeks), } \\
\text { median (IQR) }\end{array}$ & $38.86(38-40)$ & $\begin{array}{l}39.29(38.14- \\
40.21)\end{array}$ & $\begin{array}{l}39.29(38.43- \\
40.14)\end{array}$ & $\begin{array}{l}39.71(38.71- \\
40.29)\end{array}$ & 0.001 \\
\hline $\begin{array}{l}\text { Birthweight (grams), mean } \\
+/- \text { SD }\end{array}$ & $3289.22+/-500.71$ & $3307.08+/-510.77$ & $3358.20+/-475.59$ & $3434.51+/-468.78$ & 0.034 \\
\hline Apgar 1-minute, median (IQR) & $9(9-9)$ & $9(8-9)$ & $9(8-9)$ & $9(9-9)$ & 0.023 \\
\hline Apgar 1-minute, binary & & & & & 0.082 \\
\hline Less than 7 & $12(7.2 \%)$ & $25(15.2 \%)$ & $16(9.7 \%)$ & $14(8.5 \%)$ & \\
\hline More than 7 & $154(92.8 \%)$ & $139(84.8 \%)$ & $149(90.3 \%)$ & $150(91.5 \%)$ & \\
\hline Apgar 5 minute, median (IQR) & $9(9-9)$ & $9(9-9)$ & $9(9-9)$ & $9(9-9)$ & 0.591 \\
\hline Apgar 5-minute, binary & & & & & 0.04 \\
\hline Less than 7 & $1(0.6 \%)$ & $7(4.3 \%)$ & $1(0.6 \%)$ & $2(1.2 \%)$ & \\
\hline More than 7 & $165(99.4 \%)$ & $157(95.7 \%)$ & $164(99.4 \%)$ & $162(98.8 \%)$ & \\
\hline Neonatal resuscitation & & & & & 0.014 \\
\hline No & $60(36.1 \%)$ & $36(22.2 \%)$ & $56(33.9 \%)$ & $61(37.2 \%)$ & \\
\hline Yes & $106(63.9 \%)$ & $126(76.8 \%)$ & $108(65.5 \%)$ & $103(62.8 \%)$ & \\
\hline $\begin{array}{l}\text { Tactile } \\
\text { stimulation }\end{array}$ & & & & & 0.018 \\
\hline \multicolumn{6}{|l|}{ (resuscitation) } \\
\hline No & $61(36.7 \%)$ & $39(24.1 \%)$ & $58(35.4 \%)$ & $65(39.6 \%)$ & \\
\hline Yes & $105(63.3 \%)$ & $123(75.9 \%)$ & $106(64.6 \%)$ & $99(60.4 \%)$ & \\
\hline $\begin{array}{l}\text { Continuous positive } \\
\text { airway }\end{array}$ & & & & & 0.047 \\
\hline \multicolumn{6}{|l|}{$\begin{array}{l}\text { pressure (CPAP) } \\
\text { (resuscitation) }\end{array}$} \\
\hline No & $152(91.6 \%)$ & $134(82.7 \%)$ & $148(90.2 \%)$ & $148(90.2 \%)$ & \\
\hline Yes & $14(8.4 \%)$ & $28(17.3 \%)$ & $16(9.8 \%)$ & $16(9.8 \%)$ & \\
\hline
\end{tabular}




\begin{tabular}{|c|c|c|c|c|c|}
\hline $\begin{array}{l}\text { Intermittent positive } \\
\text { pressure ventilation (IPPV) } \\
\text { (resuscitation) }\end{array}$ & & & & & 0.001 \\
\hline No & $155(93.4 \%)$ & $135(83.3 \%)$ & $152(92.7 \%)$ & $156(95.1 \%)$ & \\
\hline Yes & $11(6.6 \%)$ & $27(16.7 \%)$ & $12(7.3 \%)$ & $8(4.9 \%)$ & \\
\hline $\begin{array}{l}\text { Oxygen therapy } \\
\text { (resuscitation) }\end{array}$ & & & & & 0.151 \\
\hline No & $166(100.0 \%)$ & $160(98.8 \%)$ & $163(99.4 \%)$ & $164(100.0 \%)$ & \\
\hline Yes & $0(0.0 \%)$ & $2(1.2 \%)$ & $1(0.6 \%)$ & $0(0.0)$ & \\
\hline Dextrose (resuscitation) & & & & & 0.253 \\
\hline No & $166(100.0 \%)$ & $161(99.4 \%)$ & $164(100.0 \%)$ & $164(100.0 \%)$ & \\
\hline Yes & $0(0.0 \%)$ & $1(0.6 \%)$ & $0(0.0)$ & $0(0.0)$ & \\
\hline $\begin{array}{l}\text { Volume } \\
\text { expander (resuscitation) }\end{array}$ & & & & & 0.483 \\
\hline No & $166(100.0 \%)$ & $161(99.4 \%)$ & $163(99.4 \%)$ & $164(100.0 \%)$ & \\
\hline Yes & $0(0.0 \%)$ & $1(0.6 \%)$ & $1(0.6 \%)$ & $0(0.0)$ & \\
\hline $\begin{array}{l}\text { External cardiac massage and } \\
\text { ventilation (resuscitation) }\end{array}$ & & & & & 0.496 \\
\hline No & $166(100.0 \%)$ & $161(99.4 \%)$ & $163(99.4 \%)$ & $164(100.0 \%)$ & \\
\hline Yes & $0(0.0 \%)$ & $1(0.6 \%)$ & $1(0.6 \%)$ & $0(0.0)$ & \\
\hline Neonatal characteristics & $\begin{array}{l}\text { DDI category } 1 \\
<155 \text { mins } \\
(n=166)\end{array}$ & $\begin{array}{l}\text { DDI category } 2 \\
155-314 \text { mins } \\
(n=164)\end{array}$ & $\begin{array}{l}\text { DDI category } 3 \\
314-526.5 \text { mins } \\
(n=165)\end{array}$ & $\begin{array}{l}\text { DDI category } 4 \\
>526.5 \text { mins } \\
(n=164)\end{array}$ & P-value \\
\hline $\begin{array}{l}\text { Transfer to tertiary care } \\
\text { centre }\end{array}$ & & & & & 0.781 \\
\hline No & $165(99.4 \%)$ & $162(98.8 \%)$ & $162(98.2 \%)$ & $162(98.8 \%)$ & \\
\hline Yes & $1(0.6 \%)$ & $2(1.2 \%)$ & $3(1.8 \%)$ & $2(1.2 \%)$ & \\
\hline $\begin{array}{l}\text { Admission to special care } \\
\text { nursery }\end{array}$ & & & & & 0.972 \\
\hline No & $130(78.3 \%)$ & $129(78.7 \%)$ & $130(78.8 \%)$ & $125(76.2 \%)$ & \\
\hline Yes & $36(21.7 \%)$ & $35(21.3 \%)$ & $35(21.2 \%)$ & $39(23.8 \%)$ & \\
\hline $\begin{array}{l}\text { Respiratory issues (SCN } \\
\text { admission) }\end{array}$ & & & & & 0.508 \\
\hline No & $32(88.9 \%)$ & $30(14.3 \%)$ & $30(85.7 \%)$ & $30(76.9 \%)$ & \\
\hline Yes & $4(11.1 \%)$ & $5(14.3 \%)$ & $5(14.3 \%)$ & $9(23.1 \%)$ & \\
\hline Feeding difficulties & & & & & 0.786 \\
\hline No & $100(90.1 \%)$ & $104(91.2 \%)$ & $106(87.6 \%)$ & $103(88.0 \%)$ & \\
\hline Yes & $11(9.9 \%)$ & $10(8.8 \%)$ & $15(12.4 \%)$ & $14(12.0 \%)$ & \\
\hline
\end{tabular}

DDI category (Table 3). Other significant predictors of neonatal resuscitation included in the multivariate model were mode of delivery, route of morphine administration and birthweight.

With regards to route of morphine administration, the subcutaneous route was shown to have statistically lowered odds (OR 0.22; 95\% $\mathrm{Cl} 0.068-0.69 \mathrm{P}=0.009$ ) of requiring resuscitation compared to the intramuscular route (reference category). There were no significant differences with regards to the intravenous route compared to the intramuscular route.

\section{Tactile stimulation}

Neonates born in the second DDI category had a
2 -fold increase in requirement for tactile stimulation compared to the longest DDI category (OR 2.05; 95\% Cl 1.20-3.51 $\mathrm{P}=0.009$ ). There were no statistically significant increases in odds in either the first $(P=0.66)$ or third $(P=0.79)$ DDI category compared to the longest DDI category (Table 3 ). Other significant predictors of requirement for tactile stimulation after birth included in the multivariate model were mode of delivery, route of morphine administration and birthweight.

With regards to route of morphine administration, the subcutaneous route was shown to have statistically lowered odds (OR 0.24; 95\% Cl 0.08-0.77 P = 0.016) of requiring tactile stimulation compared to the intramuscular route (reference category). There were no 


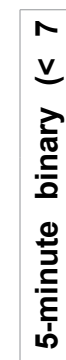

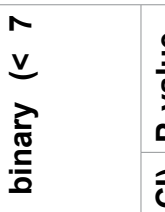

\&

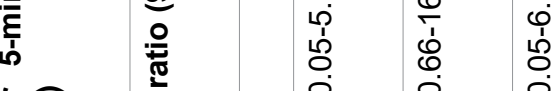

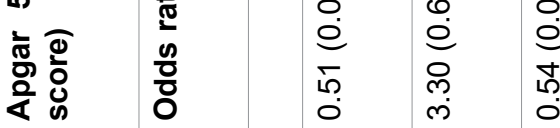

离

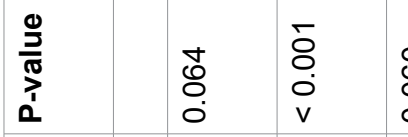

\begin{tabular}{llll}
0 & \\
\hdashline & 8 & $\overline{8}$ \\
0 & & 0 & 0 \\
0 & 0
\end{tabular}

\begin{tabular}{ll|l}
5 & 8 & 9 \\
0 & 0 & 0 \\
0
\end{tabular}

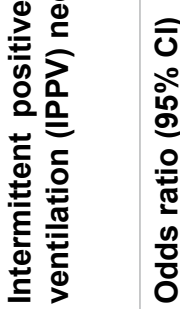

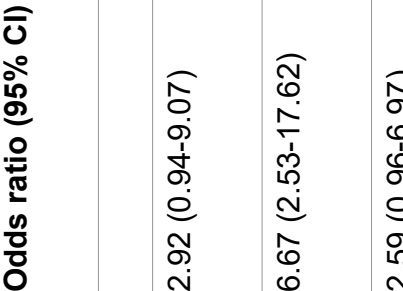

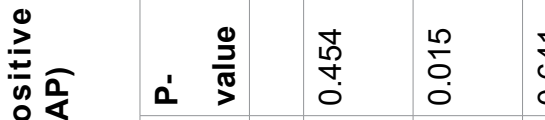

워

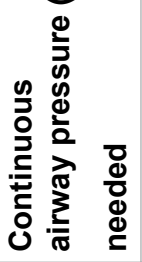

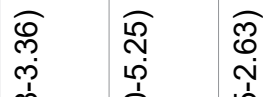

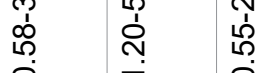

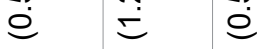

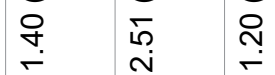

종

芒

¿

$\stackrel{\infty}{\stackrel{0}{~}} \stackrel{0}{\sim}$

\begin{tabular}{ll|l}
$\infty$ & 0 \\
0 & 0 \\
0 & 0 \\
0 & 0
\end{tabular}

竞

\begin{tabular}{|c|c|c|c|c|c|c|c|c|}
\hline ষ & 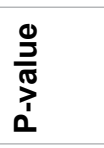 & $\begin{array}{l}\infty \\
\stackrel{0}{0} \\
0 \\
0\end{array}$ & $\begin{array}{l}8 \\
0 \\
0\end{array}$ & 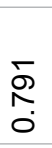 & . & $\begin{array}{l}\infty \\
\infty \\
\check{0} \\
0\end{array}$ & $\begin{array}{l}\overline{8} \\
\dot{0} \\
\text { v }\end{array}$ & , \\
\hline 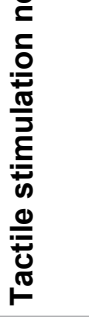 & 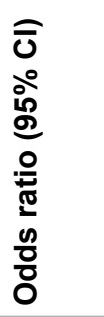 & 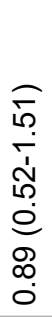 & 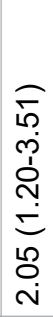 & 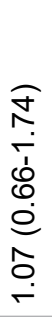 & - & 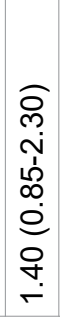 & 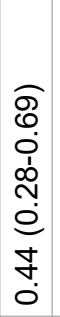 & $\begin{array}{c}- \\
-\end{array}$ \\
\hline$\frac{\sigma}{0}$ & i. $\frac{\frac{0}{5}}{\frac{5}{\nu}}$ & 疍 & : & 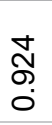 & , & $\begin{array}{l}0 \\
\tilde{ల} \\
\dddot{0} \\
0\end{array}$ & $\overline{8}$ & 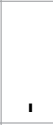 \\
\hline 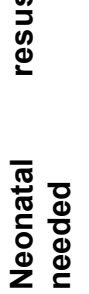 & 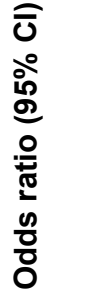 & 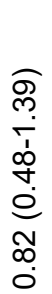 & 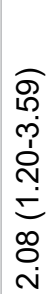 & 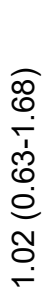 & - & 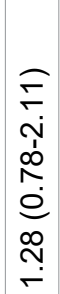 & 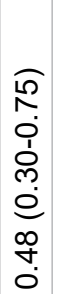 & ${ }_{-}$ \\
\hline
\end{tabular}

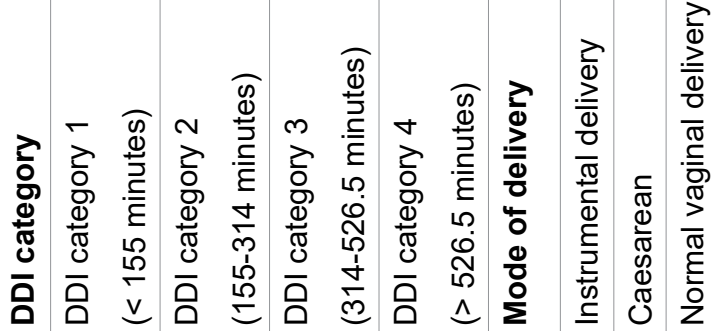

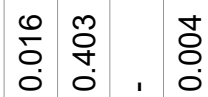

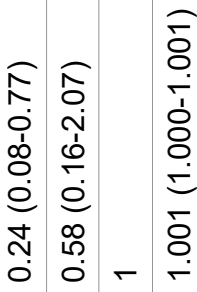

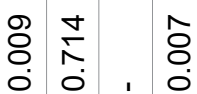

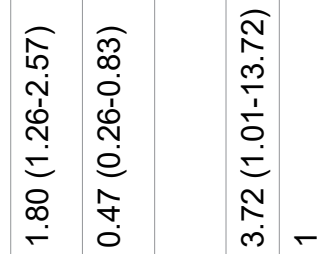

o

$\begin{array}{ll}0 & 0 \\ 0 & 1 \\ 0 & 0 \\ 0 & 0\end{array}$

$\dot{2}=$

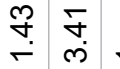

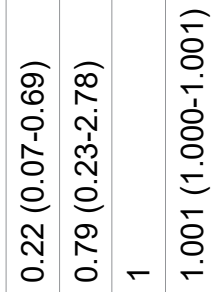

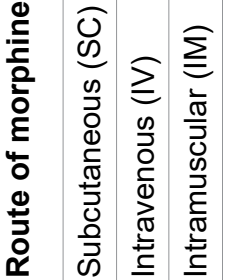



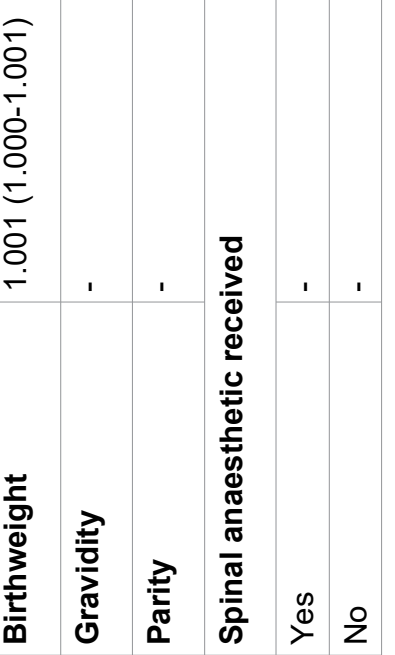


significant differences with regards to the intravenous route compared to the intramuscular route.

\section{Continuous positive airway pressure (CPAP)}

Neonates in the second DDI category had 2.5 times the odds of requiring CPAP at birth compared to the longest DDI category (OR 2.51; 95\% Cl 1.20-5.25 P = $0.015)$. There were no significant increases in odds in either the first or third DDI category compared to the longest DDI category. Other significant predictors for requirement for CPAP included in the multivariate model were mode of delivery.

\section{Intermittent positive pressure ventilation (IPPV)}

Neonates in the second DDI category had 6.7 times the odds of requiring IPPV at birth compared to the longest DDI category (OR 6.67; 95\% Cl 2.53-17.62 P< $0.001)$. There were no significant increases in odds in either the first $(P=0.064)$ or third $(P=0.060)$ DDI category compared to the longest DDI category.

\section{Discussion}

This retrospective cohort study of a large group of patients receiving morphine during labour at The Northern Hospital demonstrate a trend towards a significantly increased need for neonatal resuscitation, when the time between morphine dose and birth of the neonate falls between 155 and 314 minutes (dosedelivery interval category 2 ). This was not demonstrated by other measures of adverse neonatal outcomes.

Through our multivariate analysis, we found significantly higher odds for requiring neonatal resuscitation and in particular methods of resuscitation such as tactile stimulation, continuous positive airway pressure (CPAP) and intermittent positive pressure ventilation (IPPV) when the dose-delivery interval (DDI) was between 155 minutes and 314 minutes, compared to the longest DDI category (> 526.5 minutes). Surprisingly, this finding was not observed in either the shortest dose-delivery interval category ( $<155$ minutes) or the third DDI category (314-526.5 minutes) compared to the longest DDI category for any form of neonatal resuscitation.

The importance of dose-delivery interval is perhaps best demonstrated by studies involving use of a much more well researched labour opioid, 'pethidine'. A variety of studies have found a correlation between shorter dose-delivery intervals and an increased incidence of adverse neonatal outcomes; however, this may not necessarily be a linear relationship. One such study found the interval to sustained respiration was longer when the dose-delivery interval was 2-3 hours compared to when it was less than one hour [6]. One randomised controlled trial found a significantly higher occurrence of $<7$ Apgar scores at both 1-minute and 5-minute compared to placebo when the dose-delivery interval was 2 hours [7]. Another separate study that separated dose-delivery interval into a 'short' and 'long' dichotomous format found the shorter DDI category (1.15.3 hours) were more likely to demonstrate depressed neurobehavior compared to the longer DDI category (8.1-9.9 hours) [3,8-12]. This presents the possibility of a non-linear relationship being present with regards to the dose-delivery interval of opioid analgesia and the potential to cause adverse neonatal outcomes.

A majority of literature regarding the use of morphine during labour is focused around randomised control trials, comparing morphine to pethidine. Our study is one of the few studies to observe a morphine derivative alone and to relate and compare the incidence of adverse neonatal outcomes amongst varying dose-delivery intervals (DDI). Our study also features a significantly larger sample size in comparison to a majority of the literature pertaining to this area.

\section{Neonatal resuscitation}

One previous study found a significantly higher requirement for neonatal resuscitation when the concentration of morphine in the cord venous plasma was higher [4]. While no direct correlation was made between need for resuscitation and dose-delivery interval, it was found that shorter dose-delivery interval resulted in significantly higher concentration of morphine in cord venous plasma, suggesting that shorter dose-delivery intervals could result in an increased predisposition to requiring neonatal resuscitation. Our research, while not featuring cord venous plasma findings, reflects somewhat of a similar finding. We differ from past research, by separating dose-delivery interval into categories rather than treating it as a continuous variable.

Our findings suggest that the relationship between dose-delivery interval and requirement for resuscitation is not linear, instead it may resemble a U-shaped relationship. The second dose-delivery interval category (155-314 minutes) was found to consistently exhibit a significantly higher odd of requiring resuscitation, however, this same finding was not reflected in the shortest dose-delivery category (< 155 minutes). This reflects similar findings to those observed in studies involving pethidine, that perhaps there is a specific timeframe for dose-delivery interval that is much more likely to have an adverse impact on neonatal outcomes. While in pethidine this timeframe may be $120-180$ minutes (2-3 hrs), with morphine it may be slightly more delayed at 155-314 minutes.

In terms of trying to explain this finding, further understanding of the pharmacokinetics and metabolism of morphine is required. Ideally, if cord venous plasma concentrations of morphine and its metabolites were available, we would be able to better contextualise these findings. However, given the retrospective nature of this study, this was not possible. 


\section{Limitations}

There are a variety of limitations in our study, some of which have been listed above. First and foremost, our study is in the form of a retrospective analysis, hence our reliance on accurate record entry is substantial. However, we have attempted to mitigate the effects of inaccurate record entry by having a large sample size, to dilute any impact these records may have had.

With regards to confounding factors, the most apparent potential confounders would be that of other analgesia used during the labour. We have accounted for these in our study, but we have not included data with regards to the dose-delivery intervals of these particular medications.

This would be useful however may have complicated the study even further, hence were omitted.

\section{Future direction}

In the future, ideally similar research can be undertaken on different patient cohorts with a more elaborate analysis of neonatal wellbeing. An area of interest is the potential delayed neurobehavioral impacts intrapartum opioids may have; however, this would require a more reliable system of analysing breastfeeding behaviours and neurobehavioral scores. This would allow a better understanding of the delayed impact opioids such as morphine may have on the neonate.

\section{Conclusion}

In conclusion, our data suggests that the time interval between intrapartum morphine and birth can impact the likelihood of adverse neonatal outcomes. While shorter dose-delivery intervals do not necessarily lead to an increased predisposition for adverse neonatal outcomes, there may be a window period that does. Our research suggests the dose-delivery interval between 155 and 314 minutes may lead to a higher incidence of requirement for resuscitation at birth, in addition to specific types of resuscitation including tactile stimulation, continuous positive airway pressure and intermittent positive pressure ventilation.

Despite the limitations of our study, our findings are of interest and warrant further work to assess whether this dose-delivery interval window may result in an increased potential for adverse neonatal outcomes. Monitoring the dose-delivery interval of drugs such as morphine may help in anticipation and identification of higher risk neonates during the birthing process.

\section{Acknowledgements}

We acknowledge Mr Mark Tacey, our biostatistician for his help in analysing data.

\section{References}

1. Katzung BG, Masters SB, Trevor AJ (2009) Basic and clinical pharmacology. ( $11^{\text {th }}$ edn), McGraw-Hill, New York.

2. Anderson D (2011) A review of systemic opioids commonly used for labor pain relief. J Midwifery Womens Health 56: 222-239.

3. Nissen E, Widstrom AM, Lilja G, Matthiesen AS, UvnasMoberg K, et al. (1997) Effects of routinely given pethidine during labour on infants' developing breastfeeding behaviour. Effects of dose-delivery time interval and various concentrations of pethidine/norpethidine in cord plasma. Acta Paediatr 86: 201-208.

4. Rawal N, Tomlinson AJ, Gibson GJ, Sheehan TMT (2007) Umbilical cord plasma concentrations of free morphine following single-dose diamorphine analgesia and their relationship to dose-delivery time interval, Apgar scores and neonatal respiration. Eur J Obstet Gynecol Reprod Biol 133: $30-33$.

5. Fairlie FM, Marshall L, Walker JJ, Elbourne D (1999) Intramuscular opioids for maternal pain relief in labour: A randomised controlled trial comparing pethidine with diamorphine. Br J Obstet Gynaecol 106: 1181-1187.

6. Belfrage $\mathrm{P}$, Boreus LO, Hartvig $\mathrm{P}$, Irestedt L, Raabe $\mathrm{N}$ (1981) Neonatal depression after obstetrical analgesia with pethidine. The role of the injection-delivery time interval and of the plasma concentrations of pethidine and norpethidine. Acta Obstet Gynecol Scand 60: 43-49.

7. Sosa CG, Balaguer E, Alonso JG, Panizza R, Laborde A, et al. (2004) Meperidine for dystocia during the first stage of labor: A randomized controlled trial. Am J Obstet Gynecol 191: 1212-1218.

8. Gerdin E, Salmonson T, Lindberg B, Rane A (1990) Maternal kinetics of morphine during labour. J Perinat Med 18: $479-487$.

9. Tuckey JP, Prout RE, Wee MYK (2008) Prescribing intramuscular opioids for labour analgesia in consultantled maternity units: A survey of UK practice. Int $\mathrm{J}$ Obstet Anesth 17: 3-8.

10. Pacifici GM (2016) Metabolism and pharmacokinetics of morphine in neonates: A review. Clinics 71: 474-480.

11. Wee MYK, Tuckey JP, Thomas PW, Burnard S (2014) A comparison of intramuscular diamorphine and intramuscular pethidine for labour analgesia: A two-centre randomised blinded controlled trial. BJOG 121: 447-456.

12. Waldmann CS, Eason JR, Rambohul E, Hanson GC (1984) Serum morphine levels. A comparison between continuous subcutaneous infusion and continuous intravenous infusion in postoperative patients. Anaesthesia 39: 768-771. 